\title{
Hospitalizaciones por insuficiencia cardiaca descompensada en España
}

Macaya Miguel C, Bover Freire R. Hospitalizaciones por insuficiencia cardiaca descompensada en España. An Med Interna (Madrid) 2008; 25: 253-255.

La insuficiencia cardiaca continúa siendo en nuestro entorno, y de forma universal, uno de los principales retos diagnósticos en el ámbito de las enfermedades cardiacas. Considerada por diversas voces autorizadas como una auténtica epidemia cardiovascular del siglo XXI (1), no conocemos adecuadamente su epidemiología, las implicaciones clínicas de sus diferentes presentaciones (insuficiencia cardiaca sistólica versus diastólica), sus matices diagnósticos más complejos, ni el abordaje global que individualmente aplicado en las fases de descompensación pudiera mejorar su pronóstico final. Es por ello de gran interés el estudio publicado por Hermida y cols. (2) de los factores epidemiológicos que pueden estar asociados a la hospitalización motivada por su descompensación. Los autores realizan un análisis retrospectivo de 209 pacientes ingresados por insuficiencia cardíaca descompensada en el servicio de Medicina Interna de un hospital de tercer nivel. En su serie, la mayoría de las descompensaciones se producen en el seno de infecciones respiratorias, y se identifican como sus principales factores de riesgo los estadios funcionales de la NYHA o de la Cruz Roja más avanzados, así como la presencia de demencia o ictus.

La epidemiología de la insuficiencia cardiaca es difícil de investigar. Ello se debe, entre otros motivos, a las propias dificultades de su diagnóstico, eminentemente clínico, fácil de confundir con otras patologías prevalentes como la enfermedad pulmonar obstructiva crónica, o con perfiles clínicos que pueden ocasionar una sintomatología similar, como la obesidad. Varios estudios poblacionales bien diseñados han permitido conocer su incidencia y prevalencia dentro y fuera de nuestras fronteras $(3,4)$. Por otro lado, existen numerosos registros, algunos de ellos con una amplia muestra de pacientes, que nos ofrecen información relevante de sus principales características clínicas, así como de las propiedades diferenciales de diversos subgrupos de pacientes (5-9).

A la hora de analizar las hospitalizaciones por insuficiencia cardiaca en nuestro entorno debemos tener en cuenta varios aspectos relevantes. En primer lugar, estas puede producirse en hospitales de diferente nivel tecnológico, así como en distintos servicios clínicos (Medicina Interna, Geriatría o Cardiología, principalmente) entre los que, en ocasiones, no existe suficiente coordinación asistencial; ambos factores pueden condicionar de forma notable las posibilidades diagnósticas y terapéuticas disponibles, así como las finalmente empleadas. Si la situación clínica del paciente lo permite deben valorarse otros modelos alternativos a la hospitalización clásica, como son las Unidades de Corta Estancia o permanencias breves en los propios servicios de Urgencias. Por otro lado, hay que diferenciar entre los pacientes en los que la insuficiencia cardíaca es el motivo principal de ingreso de aquellos que presentan secundariamente la descompensación clínica durante una hospitalización por otra causa. Lamentablemente, esta diferenciación, al igual que la definición del tipo de insuficiencia cardíaca y la causa de su descompensación, no queda siempre suficientemente clara en los informes de alta, ocasionando errores en la codificación de los episodios y, consecuentemente, en su análisis posterior. Hubiese sido deseable en el artículo de Hermida y cols. conocer el número de ingresos durante ese año, totales y por insuficiencia cardiaca, en todo el hospital y en el servicio de Medicina Interna, al igual que en otras áreas de hospitalización. La calidad de la codificación de los diagnósticos adquiere especial relevancia dada la selección retrospectiva de los pacientes incluidos en su estudio. En segundo lugar, hay que considerar los diversos condicionantes fisiopatológicos de la insuficiencia cardiaca. Es necesario diferenciar y analizar por separado los factores que participan en la propia etiología del síndrome (causas predisponentes o factores de riesgo), las alteraciones cardiovasculares estructurales o funcionales que lo originan (causas determinantes), y los factores que provocan la descompensación de una situación de estabilidad en pacientes con o sin diagnóstico previo de insuficiencia cardiaca (causas precipitantes, cardiacas o extracardiacas) (10); igualmente, es preciso analizar con cautela diversas situaciones clínicas concomitantes (anemia, infecciones respiratorias e insuficiencia renal, entre otras) que, aunque con frecuencia son la causa de la descompensación, en ocasiones son meros acompañantes o 
incluso consecuencia de la misma. Debe analizarse con el máximo rigor metodológico posible el papel que juegan en la descompensación factores como la hipertensión arterial o la cardiopatía isquémica; la relación existente entre ambas es bien conocida, pero su participación como causas predisponentes, determinantes o precipitantes puede ser sustancialmente distinta. El diagnóstico etiológico correcto es especialmente importante, ya que puede permitir la realización de pruebas complementarias o actitudes terapéuticas específicas durante la propia hospitalización (coronariografía y angioplastia, cirugía coronaria o valvular, o implante de dispositivos, entre otros). La creciente implantación de Unidades de Insuficiencia Cardíaca en nuestro país permitirá reducir notablemente las hospitalizaciones, gracias a la detección y tratamiento precoz de las causas precipitantes de la descompensación. La incorporación de la telemedicina puede del mismo modo mejorar notablemente, frente al abordaje convencional, el diagnóstico eficaz de la descompensación en sus fases más precoces. En tercer lugar, la descompensación de la insuficiencia cardíaca no se manifiesta de forma uniforme, sino que presenta un amplio espectro clínico con diferentes implicaciones pronósticas: insuficiencia cardíaca aguda descompensada (de novo o descompensación de una insuficiencia cardíaca crónica), insuficiencia cardíaca aguda hipertensiva, edema agudo de pulmón, shock cardiogénico e insuficiencia cardíaca con alto gasto (11). Sería deseable utilizar esta clasificación, recomendada por las sociedades internacionales, para poder profundizar en el conocimiento de las características propias diferenciales de cada una de ellas. Todos estos y otros factores dificultan el estudio de las características y condicionantes propios de la hospitalización en los pacientes con insuficiencia cardíaca.

El abordaje diagnóstico de la insuficiencia cardíaca reviste igualmente aspectos aún no resueltos y no exentos de complejidad. La Sociedad Europea de Cardiología establece en sus recomendaciones que este debe fundamentarse en la presencia de los síntomas clínicos característicos del síndrome, acompañados de la evidencia objetiva (preferiblemente por ecocardiografía) de la existencia de disfunción cardíaca sistólica y/o diastólica. No hay que olvidar la ayuda diagnóstica que suponen otras pruebas complementarias, como el electrocardiograma (cuya normalidad prácticamente excluye el diagnóstico), la radiografía de tórax, los péptidos natriuréticos (BNP y NT-proBNP) y las diversas pruebas no invasivas o cruentas de detección de isquemia, entre otras. La ecocardiografía constituye, por lo tanto, uno de los pilares diagnósticos fundamentales, siendo al mismo tiempo esencial para la determinación certera de la cardiopatía subyacente. En el artículo de Hermida y cols., (2) el $30,6 \%$ de los pacientes no disponen de un ecocardiograma durante la hospitalización, cifra análoga a la que encontramos en otras series semejantes de nuestro entorno (12). Este hecho, especialmente preocupante en un hospital de tercer nivel, puede poner en duda la fiabilidad del diagnóstico en alguno de estos pacientes, y debe llevar a la reflexión sobre la necesidad de promover la solicitud de dicha prueba durante la hospitalización, así como el compromiso necesario por parte de los servicios de Cardiología de ofertar una mayor accesibilidad a la misma en un tiempo razonable. El desarrollo y la proliferación de los nuevos equipos portáti- les de ecocardiografía permitirá en un futuro próximo "vulgarizar" aún más la técnica, posibilitando incluso la realización de una exploración básica por otros especialistas médicos. Hubiese sido igualmente deseable conocer las características del electrocardiograma de los pacientes incluidos, ya que sus alteraciones, como la fibrilación auricular, tienen un papel pronóstico relevante (13). De forma complementaria, los péptidos natriuréticos han demostrado aportar en la insuficiencia cardíaca una importante información diagnóstica y pronóstica (14), que queda ensombrecida por la baja disponibilidad de su determinación en nuestro entorno.

Finalmente, el análisis de los aspectos pronósticos (mortalidad) puede verse afectado en gran medida por la diferente situación y gravedad clínica de los pacientes al ingreso, el lugar de hospitalización y las diversas maniobras diagnósticas y terapéuticas empleadas, no siendo posible obtener datos concluyentes al respecto sin un análisis conjunto de todos estos factores $(15,16)$. Resulta igualmente relevante el conocimiento de la causa de la muerte, diferenciando aquellas de origen cardiovascular del resto.

La información derivada del artículo de Hermida y cols. (2), presenta algunas limitaciones reseñables, algunas de ellas ya comentadas. La potencia estadística alcanzada es, a la luz de los resultados, insuficiente para llegar a conclusiones sólidas sobre algunos de los aspectos analizados, especialmente en subgrupos específicos con escaso tamaño muestral (pacientes con disfunción sistólica). Por otro lado, representa únicamente a los pacientes hospitalizados en servicios de Medicina Interna que, aunque son mayoría, presentan un perfil epidemiológico y clínico sustancialmente distinto de los ingresados en los servicios de Cardiología, más frecuentemente varones, de menor edad, con menos comorbilidades, mayor prevalencia de disfunción sistólica y mayor empleo de las técnicas diagnósticas y terapéuticas disponibles. Los anteriores condicionantes, añadidos a la naturaleza retrospectiva del análisis, pueden explicar que la proporción observada de los diversos agentes precipitantes posibles (infección respiratoria, anemia, crisis hipertensiva e incumplimiento terapéutico, entre otros) sea diferente a otras series publicadas (17). Especialmente relevante es la información aportada por Hermida y cols., (2) sobre los pacientes con insuficiencia cardíaca con función sistólica conservada que son hospitalizados en nuestro entorno, ya que este es el subgrupo de pacientes en el que existe menos consenso sobre su abordaje diagnóstico y manejo clínico óptimo.

Por todo ello, los resultados observados por Hermida y cols., (2) tienen a nuestro juicio un importante valor descriptivo, más que inferencial, de los factores epidemiológicos relacionados con la descompensación de la insuficiencia cardiaca en nuestro entorno. El estudio realizado pone al mismo tiempo de manifiesto las limitaciones pendientes de resolver en el manejo diagnóstico y en el abordaje terapéutico de este complejo síndrome.

\section{MACAYA, R. BOVER FREIRE ${ }^{1}$}

Servicio de Cardiología. ${ }^{1}$ Unidad de Insuficiencia

Cardiaca. Hospital Clínico San Carlos. Madrid 


\section{Bibliografía}

1. Cleland JGF, Khand A, Clark A. The heart failure epidemic: exactly how big is it?. Eur Heart J 2001; 22: 623-6.

2. Hermida Ameijeiras A, Pazo Núñez M, de la Fuente Cid R, Lado Lado FL, Hernández Fernández L, Rodríguez López I (Grupo SANTICOR). Factores epidemiológicos asociados a la hospitalización por descompensación de la insuficiencia cardíaca. An Med Interna (Madrid) 2008; 25: 256-61.

3. Cortina A, Reguero J, Segovia E, Rodríguez Lambert JL, Cortina R, Arias JC, et al. Prevalence of heart failure in Asturias (a region in the North of Spain). Am J Cardiol 2001; 87: 1417-9.

4. Rodríguez-Artalejo F, Banegas JR, Guallar P. Epidemiología de la insuficiencia cardiaca. Rev Esp Cardiol 2004; 57: 163-70.

5. Komajda M, Follath F, Swedberg K, Cleland J, Aguilar JC, Cohen-Solal A, et al, for the Study Group on Diagnosis of the Working Group on Heart Failure of the European Society of Cardiology. The EuroHeart Failure survey programme: a survey on the quality of care among patients with heart failure in Europe. Part 2. Treatment. Eur Heart J 2003; 24: 464-74.

6. Nieminen MS, Brutsaert D, Dickstein K, Drexler H, Follath F, Harjola VP, et al. EuroHeart Failure Survey II (EHFS II): a survey on hospitalized acute heart failure patients: description of population. Eur Heart J 2006; 27: 2725-36.

7. Adams KF Jr, Fonarow GC, Emerman CL, LeJemtel TH, Costanzo MR, Abraham WT, Berkowitz RL, Galvao M, Horton DP, ADHERE Scientific Advisory Committee and Investigators. Characteristics and outcomes of patients hospitalized for heart failure in the United States: rationale, design, and preliminary observations from the first 100,000 cases in the acute decompensated heart failure national registry (ADHERE). Am Heart J 2005; 149: 209-16.

8. Gheorghiade M, Abraham WT, Albert NM, Greenberg BH, O'Connor $\mathrm{CM}$, She L, et al. Systolic blood pressure at admission, clinical characteristics, and outcomes in patients hospitalized with acute heart failure. JAMA 2006; 296: 2217-26.

9. Cleland JG, Cohen-Solal A, Cosín Aguilar J, Dietz R, Eastaugh J, Follath F, et al. Management of heart failure in primary care (the
IMPROVEMENT of Heart Failure Programme): an international survey. Lancet 2002; 360: 1631-9.

10. Segovia J, Alonso-Pulpón L, Peraira R, Silva L. Etiología y evaluación diagnóstica en la insuficiencia cardíaca. Rev Esp Cardiol 2004; 57: 250-59.

11. Swedberg K, Cleland J, Dargie H, Drexler H, Follath F, Komajda M, et al. Guidelines for the diagnosis and treatment of chronic heart failure: executive summary (update 2005): the Task Force for the Diagnosis and Treatment of Chronic Heart Failure of the European Society of Cardiology. Eur Heart J 2005; 26: 1115-40.

12. Otero-Raviña F, Grigorian-Shamagian L, Fransi-Galiana L, Názara-Otero C, Fernández-Villaverde JM, del Alamo-Alonso A, et al; GALICAP study investigators. Estudio gallego de insuficiencia cardiaca en atención primaria (estudio GALICAP). Rev Esp Cardiol 2007; 60: 373-83.

13. Olsson LG, Swedberg K, Ducharme A, Granger CB, Michelson EL, McMurray JJ, et al; CHARM Investigators. Atrial fibrillation and risk of clinical events in chronic heart failure with and without left ventricular systolic dysfunction: results from the Candesartan in Heart failureAssessment of Reduction in Mortality and morbidity (CHARM) program. J Am Coll Cardiol 2006; 47: 1997-2004.

14. Fonarow GC, Peacock WF, Phillips CO, Givertz MM, Lopatin M; ADHERE Scientific Advisory Committee and Investigators. Admission B-type natriuretic peptide levels and in-hospital mortality in acute decompensated heart failure. J Am Coll Cardiol 2007; 49: 1943-50.

15. Owan TE, Hodge DO, Herges RM, Jacobsen SJ, Roger VL, Redfield MM. Trends in prevalence and outcome of heart failure with preserved ejection fraction. N Engl J Med 2006; 355: 251-59.

16. Bhatia RS, Tu JV, Lee DS, Austin PC, Fang J, Haouzi A, et al. Outcome of heart failure with preserved ejection fraction in a population-based study. N Engl J Med 2006; 355: 260-9.

17. Nieminen MS, Harjola VP, Hochadel M, Drexler H, Komajda M, Brutsaert $\mathrm{D}$, et al. Gender related differences in patients presenting with acute heart failure. Results from EuroHeart Failure Survey II. Eur J Heart Fail 2008; 10: 140-8 